\title{
Photochemical $\mathrm{CO}_{2}$-to-formate/CO Conversion Catalyzed by Half- Metallocene Ir(III) Catalyst and Its Mechanistic Investigation
}

\author{
Daehan Lee, Sunghan Choi, Min Su Choe, So-Yoen Kim, Kyutai Park, Chul Hoon Kim, \\ Ho-Jin Son,* and Sang Ook Kang* \\ Department of Advanced Materials Chemistry, Korea University, Sejong 30019, Korea
}

*Correspondence and requests for materials should be addressed to

*E-mail: Ho-Jin Son: hjson@korea.ac.kr

*E-mail: Sang Ook Kang: sangok@korea.ac.kr

\begin{tabular}{|c|c|c|}
\hline \multicolumn{3}{|c|}{ Table of contents } \\
\hline Sections & Titles & pages \\
\hline Figure S1 & The schematic of the integrating sphere absorption spectrometer & $\mathrm{S} 2$ \\
\hline Figure S2 & ${ }^{1} \mathrm{H}-\mathrm{NMR}$ spectroscopic view of Cp*IrPE in DMSO- $d_{6}$ & S3 \\
\hline Figure S3 & ${ }^{1} \mathrm{H}-\mathrm{NMR}$ spectroscopic view of Cp*IrP in DMSO- $d_{6}$ & S4 \\
\hline Figure S4 & ${ }^{13} \mathrm{C}-\mathrm{NMR}$ spectroscopic view of Cp$* \mathbf{I r P E}$ in DMF- $d_{7}$ & S5 \\
\hline Figure S5 & ${ }^{13}$ C-NMR spectroscopic view of $\mathbf{C p} * \mathbf{I r P}$ in DMF- $d_{7}$ & S6 \\
\hline Figure S6 & ESI-Mass spectra of Cp*IrPE & S7 \\
\hline Figure S7 & ESI-Mass spectra of $\mathbf{C p} * \mathbf{I r P}$ & S8 \\
\hline Table S1 & Crystal data and structure refinement for $\mathbf{C p} * \mathbf{I r P E}$ & S9 \\
\hline Table S2 & Bond lengths $[\AA]$ for $\mathbf{C p}$ *IrPE & S10 \\
\hline Table S3 & Angles $\left[{ }^{\circ}\right]$ for $\mathbf{C p} * \mathbf{I r P E}$ & S11-12 \\
\hline Figure S8 & Detailed procedure for preparation of $\mathbf{I r P} / \mathrm{TiO}_{2} / \mathbf{C p} * \mathbf{I r P}$ ternary sample & S13 \\
\hline Figure S9 & $\begin{array}{l}\text { UV-vis absorption spectra of } \mathbf{C p} * \mathbf{I r P} \text { before and after adsorption process of } \mathbf{C p} * \mathbf{I r P} \text { with } \\
\mathrm{TiO}_{2} \text { particles }\end{array}$ & S14 \\
\hline Figure S10 & UV-vis absorption of IrPE and Cp*IrPE in DMF & S15 \\
\hline Figure S11 & $\begin{array}{l}\text { UV-vis absorption spectrum of reaction solution and } \mathbf{I r P} / \mathrm{TiO}_{2} / \mathbf{C p} * \mathbf{I r P} \text { powders filtered } \\
\text { before and after } 90 \mathrm{~h} \text { photolysis of hetero-ternary sample }\end{array}$ & S16 \\
\hline Figure S12 & $\begin{array}{l}{ }^{1} \mathrm{H} /{ }^{13} \mathrm{C} \text { NMR spectrum of reaction solution filtered after } 90 \mathrm{~h} \text { photolysis of hetero-ternary } \\
\text { sample }\end{array}$ & S17 \\
\hline Figure S13 & $\begin{array}{l}\text { Plots of formate and CO formation versus irradiation time for ternary hybrid system in } \\
\text { different conditions }\end{array}$ & S18 \\
\hline Figure S14 & $\begin{array}{l}\text { Formation of formate in photolysis restarted with re-addition of different components (IrPE, } \\
\text { Cp*IrPE, or BIH) after } 48 \text { h photolysis of homogeneous sample }\end{array}$ & S19 \\
\hline Figure S15 & $\begin{array}{l}{ }^{13} \mathrm{C} \text { isotope-labeled GC-MS spectra before and after irradiation in } \mathrm{CO}_{2} \text {-saturated DMF- } d_{7} \\
\text { containing } 10 \mathrm{mg} \text { of } \mathbf{I r P} / \mathrm{TiO}_{2} / \mathbf{C p} \mathbf{p}^{*} \mathbf{I r P}\end{array}$ & S20 \\
\hline Figure S16 & $\begin{array}{l}\text { Plots of formate and CO formation versus itrradiation time for ternary hybrid system with } \\
\text { variation of TEOA content ( } \mathrm{vol} \%)\end{array}$ & S21 \\
\hline Table S4 & $\begin{array}{l}\text { Results of visible-light-driven } \mathrm{CO}_{2} \text { Reduction with ternary hybrid system in different TEOA } \\
\text { vol \% conditions }\end{array}$ & S22 \\
\hline Figure S17 & Cyclic voltammograms of IrPE and Cp*IrPE in DMF & S23 \\
\hline Table S5 & Electrochemical properties of IrPE and Cp*IrPE & S23 \\
\hline
\end{tabular}




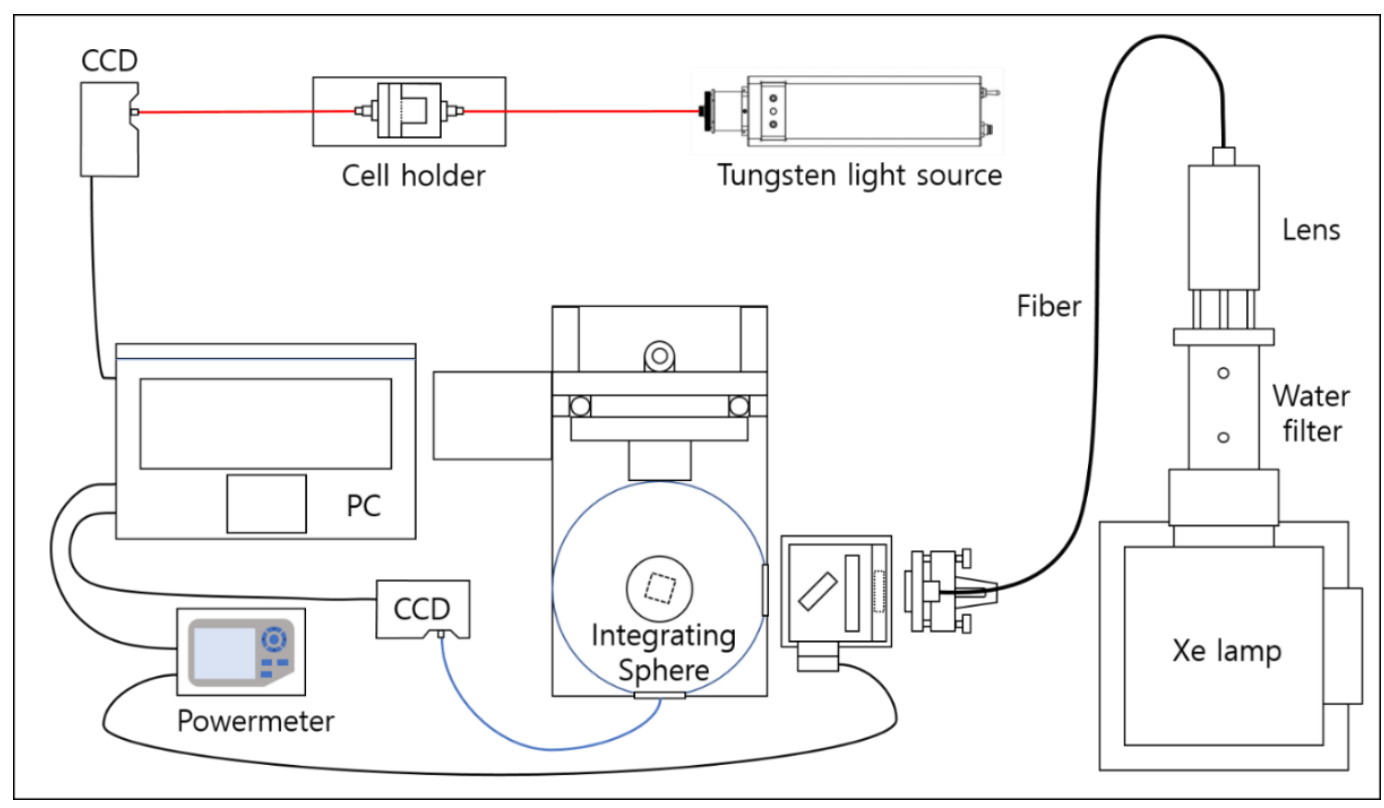

Figure S1. The schematic diagram of the integrating sphere absorption spectrometer. 


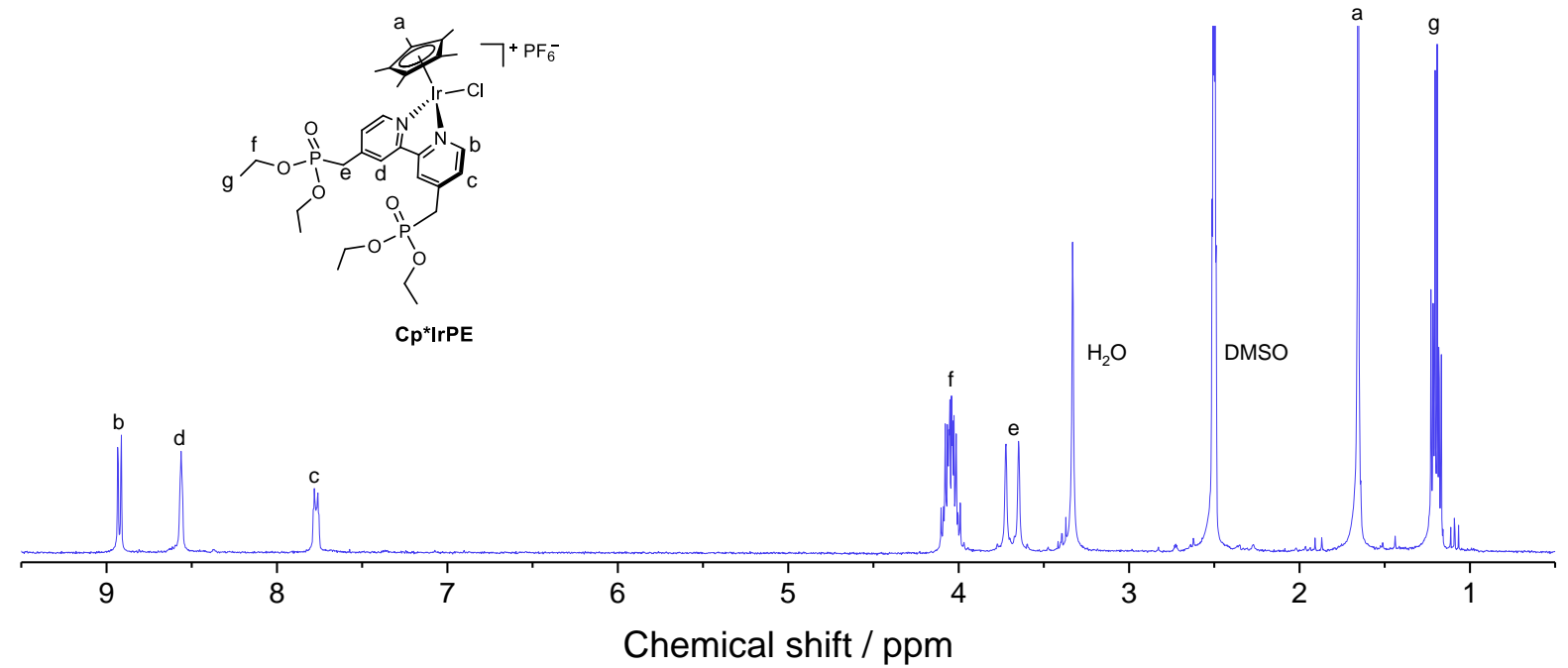

Figure S2. ${ }^{1} \mathrm{H}-\mathrm{NMR}$ spectroscopic view of Cp*IrPE in DMSO- $d_{6}$. 


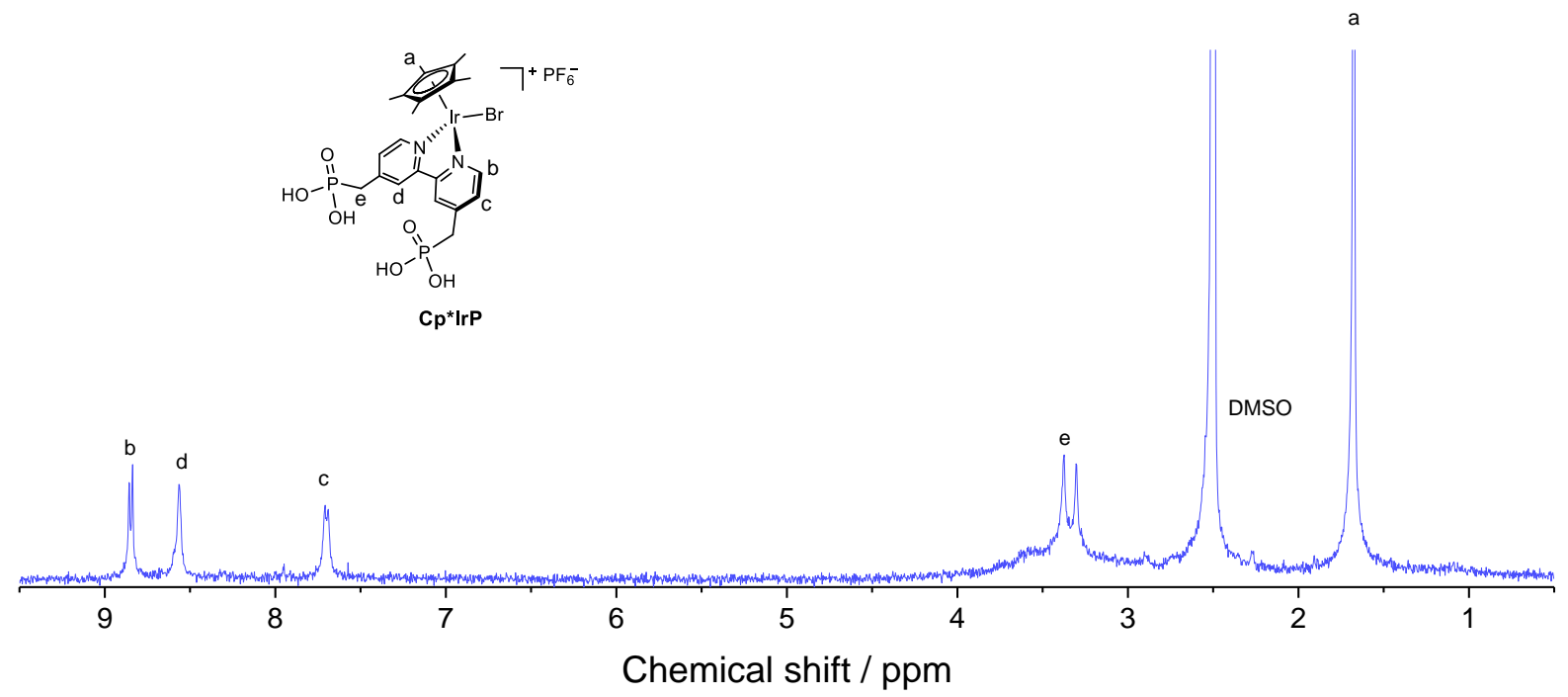

Figure S3. ${ }^{1} \mathrm{H}-\mathrm{NMR}$ spectroscopic view of Cp*IrP in DMSO- $d_{6}$. 


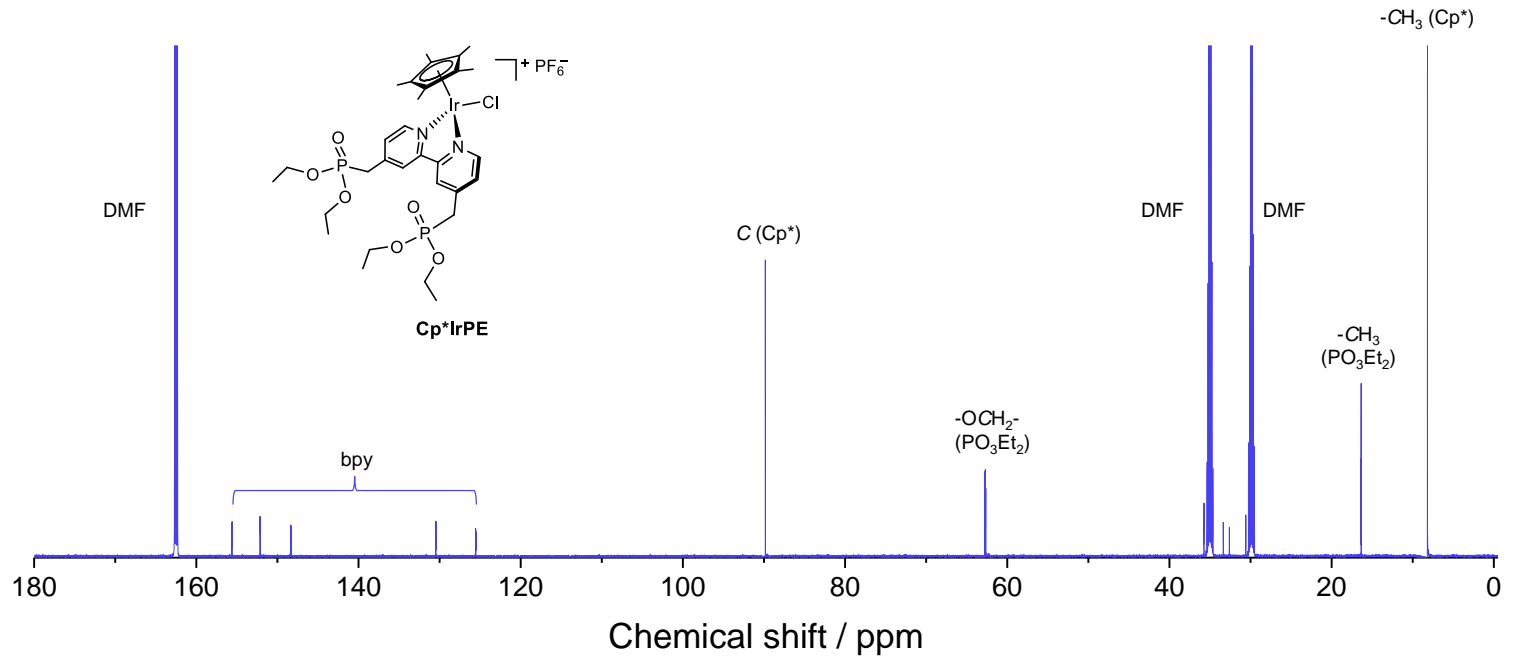

Figure S4. ${ }^{13} \mathrm{C}-\mathrm{NMR}$ spectroscopic view of $\mathbf{C p} *$ IrPE in DMF- $d_{7}$. 


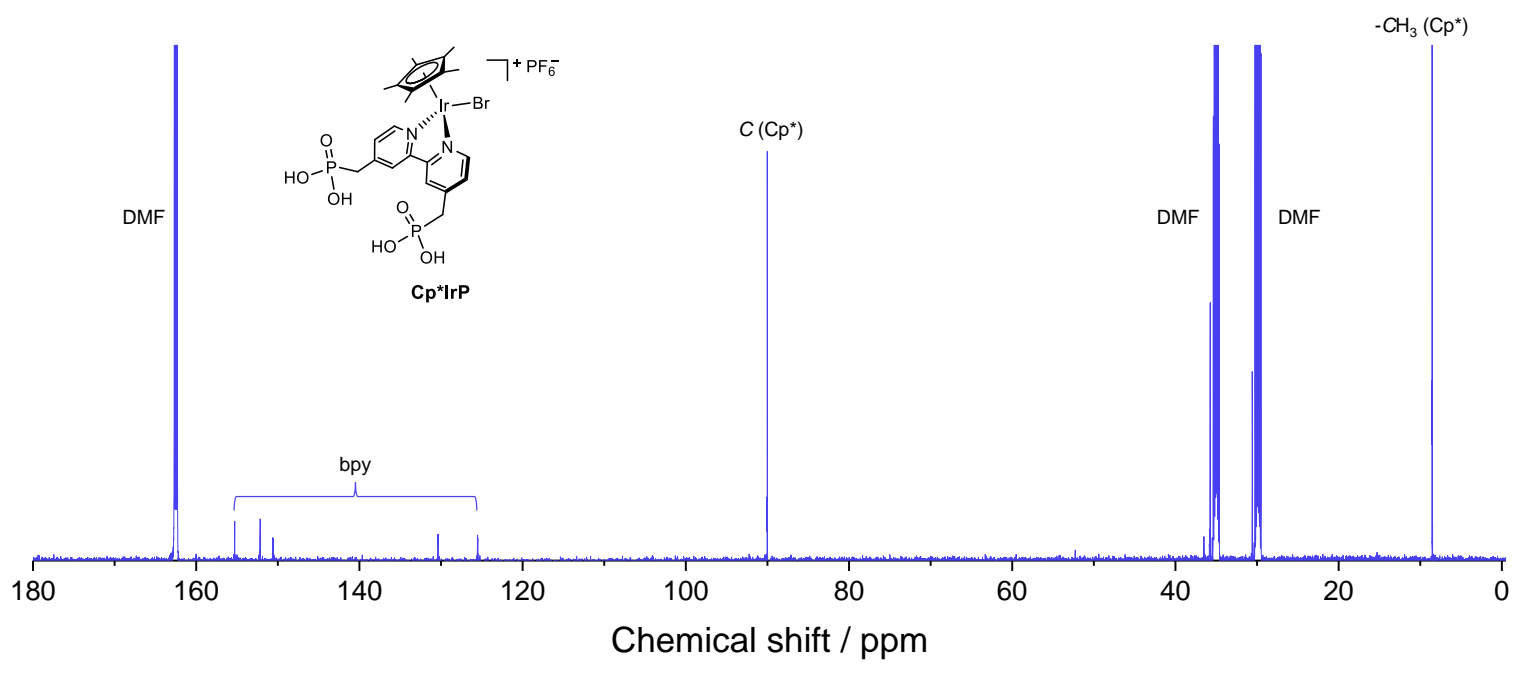

Figure S5. ${ }^{13} \mathrm{C}-\mathrm{NMR}$ spectroscopic view of Cp*IrP in DMF- $d 7$. 


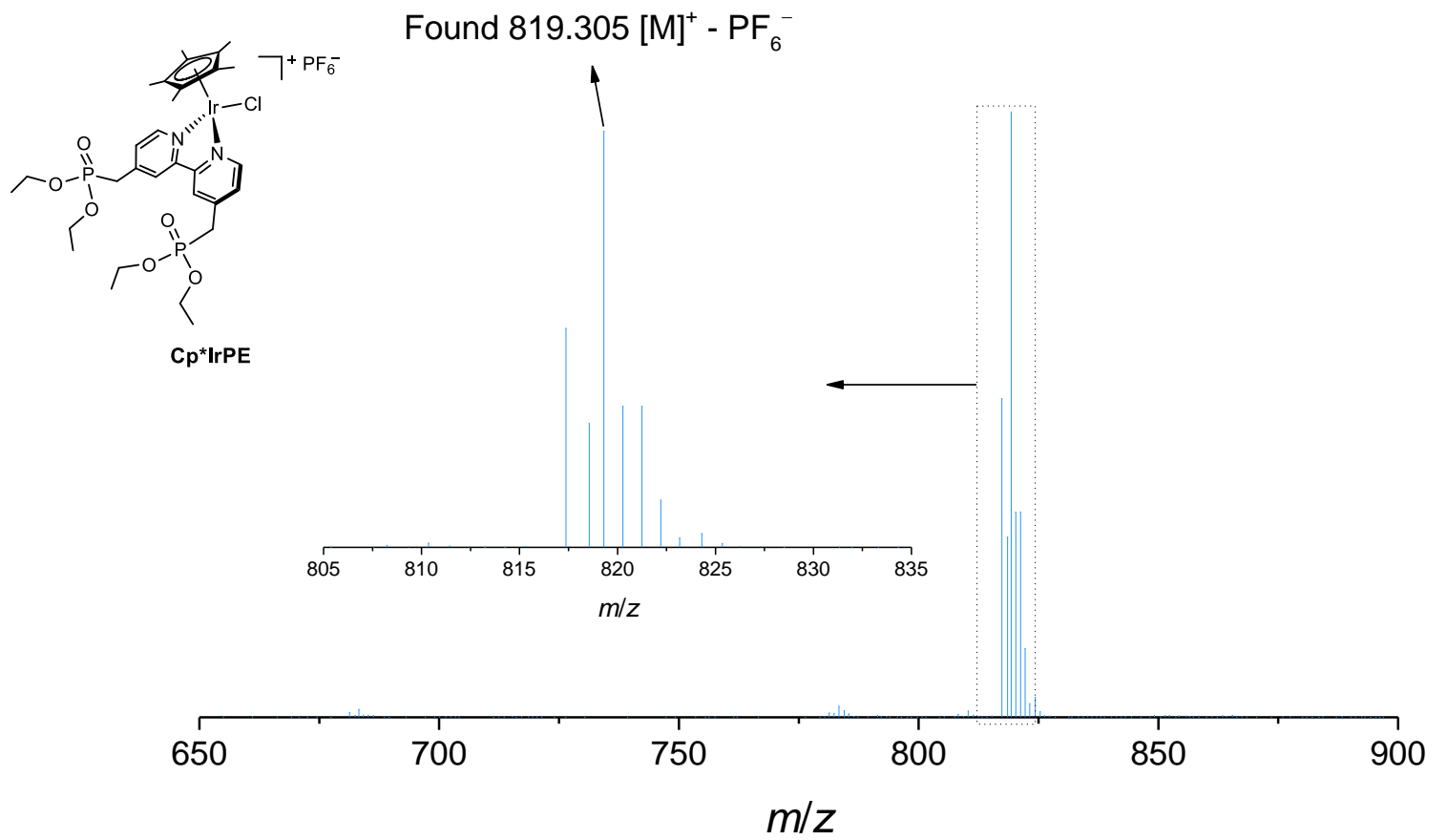

Figure S6. ESI-Mass spectra of Cp*IrPE. 


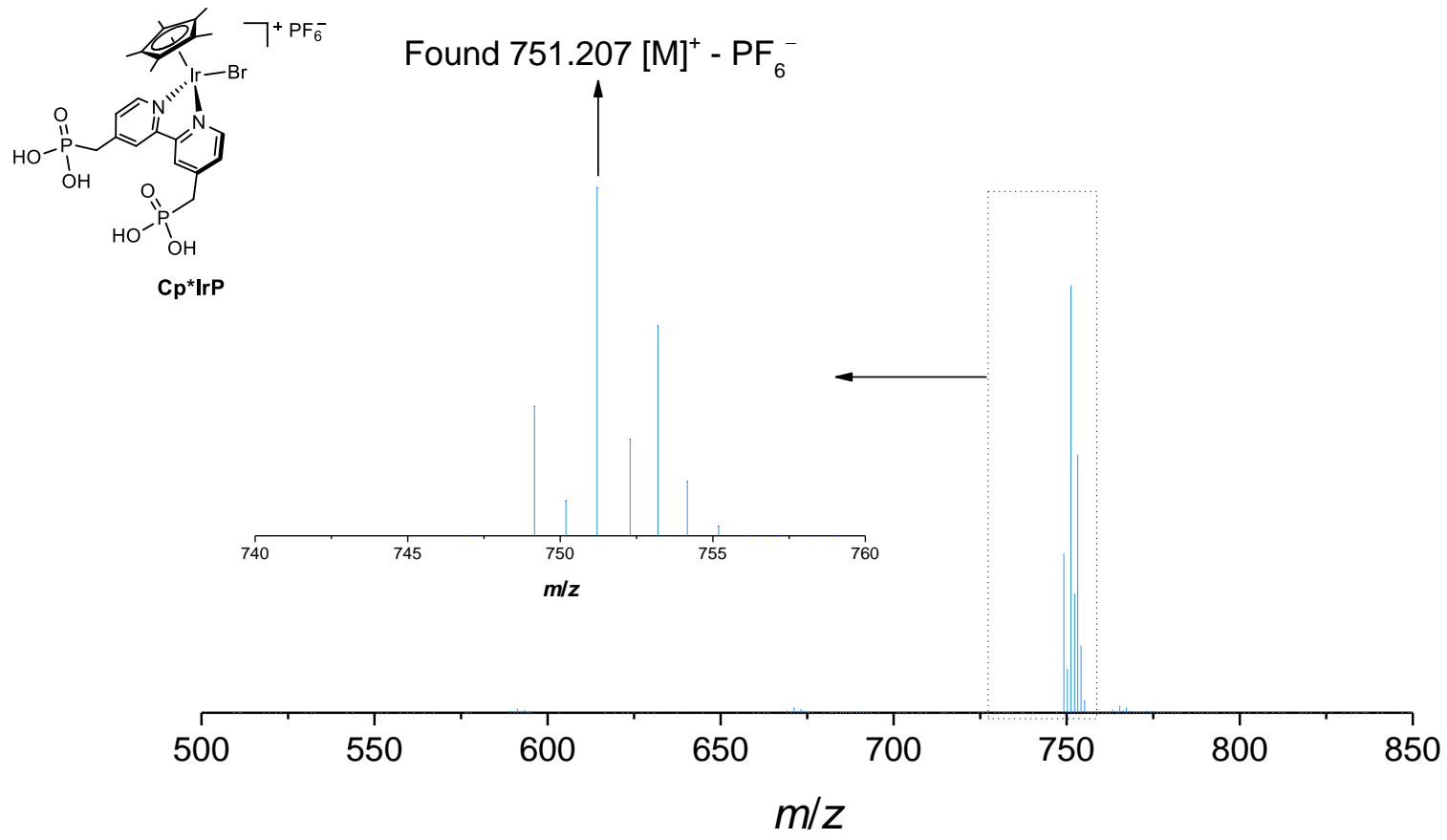

Figure S7. ESI-Mass spectra of Cp*IrP. 
Table S1. Crystal data and structure refinement for Cp*IrPE

\section{Cp*IrPE}

\begin{tabular}{|c|c|}
\hline Identification code & 2045537 \\
\hline Empirical formula & $\mathrm{C}_{32} \mathrm{H}_{41} \mathrm{~N}_{2} \mathrm{ClO}_{7} \mathrm{Ir} \mathrm{P}_{3} \mathrm{~F}_{6}$ \\
\hline Formula weight & 1000.23 \\
\hline Temperature & $296(2) \mathrm{K}$ \\
\hline Wavelength & $0.71073 \mathrm{~A}$ \\
\hline Crystal system, & Orthorhombic \\
\hline Space group & Pna $2_{1}$ \\
\hline \multirow[t]{3}{*}{ Unit cell dimensions } & $a=21.444 \AA$ \\
\hline & $b=24.045 \AA$ \\
\hline & $c=7.513 \AA$ \\
\hline Volume & $3873.7 \AA^{3}$ \\
\hline$Z, D_{\text {calc }}$ & $4,1.715 \mathrm{Mg} / \mathrm{m}^{3}$ \\
\hline$F(000)$ & 1984 \\
\hline Crystal size & $0.18 \times 0.08 \times 0.07 \mathrm{~mm}$ \\
\hline$\theta$ range for data collection & 1.694 to $30.529^{\circ}$ \\
\hline Limiting indices & $-30 \leq h \leq 30,-34 \leq k \leq 34,-10 \leq l \leq 10$ \\
\hline Reflections collected / unique & $142795 / 11772[R($ int $)=0.0511]$ \\
\hline Completeness to $\theta=25.242$ & $99.7 \%$ \\
\hline Refinement method & Full-matrix least-squares on $F^{2}$ \\
\hline Data / restraints / parameters & $11772 / 5 / 443$ \\
\hline Goodness-of-fit on $F^{2}$ & 1.163 \\
\hline Final $R$ indices $[\mathrm{I}>2 \theta(\mathrm{I})]$ & ${ }^{a} R_{1}=0.0398,{ }^{b} \mathrm{w} R_{2}=0.1016$ \\
\hline$R$ indices (all data) & ${ }^{a} R_{1}=0.0607,{ }^{b} \mathrm{w} R_{2}=0.1232$ \\
\hline Largest diff. peak and hole & 1.033 and -1.938 e. $\AA^{-3}$ \\
\hline
\end{tabular}


Table S2. Bond lengths $[\AA]$ for Cp*IrPE

\begin{tabular}{|c|c|c|c|}
\hline $\operatorname{Ir}(1)-N(2)$ & $2.09(7)$ & $\mathrm{N}(2)-\mathrm{C}(6)$ & $1.35(9)$ \\
\hline $\operatorname{Ir}(1)-\mathrm{N}(1)$ & $2.09(7)$ & $C(1)-C(2)$ & $1.37(1)$ \\
\hline $\operatorname{Ir}(1)-C(23)$ & $2.14(1)$ & $C(2)-C(3)$ & $1.40(1)$ \\
\hline $\operatorname{Ir}(1)-C(25)$ & $2.14(1)$ & $C(3)-C(4)$ & $1.40(1)$ \\
\hline $\operatorname{Ir}(1)-C(21)$ & $2.15(1)$ & $\mathrm{C}(3)-\mathrm{C}(11)$ & $1.49(1)$ \\
\hline $\operatorname{Ir}(1)-C(24)$ & $2.15(1)$ & $C(4)-C(5)$ & $1.39(1)$ \\
\hline $\operatorname{Ir}(1)-C(22)$ & $2.17(1)$ & $C(5)-C(6)$ & $1.47(1)$ \\
\hline $\operatorname{Ir}(1)-\mathrm{Cl}(1)$ & $2.38(3)$ & $C(6)-C(7)$ & $1.38(1)$ \\
\hline $\mathrm{P}(2)-\mathrm{O}(6)$ & $1.37(2)$ & $C(7)-C(8)$ & $1.37(1)$ \\
\hline $\mathrm{P}(2)-\mathrm{O}(4)$ & $1.45(9)$ & $\mathrm{C}(8)-\mathrm{C}(9)$ & $1.42(2)$ \\
\hline $\mathrm{P}(2)-\mathrm{O}(5)$ & $1.51(1)$ & $\mathrm{C}(8)-\mathrm{C}(12)$ & $1.51(1)$ \\
\hline$P(2)-C(12)$ & $1.76(2)$ & $\mathrm{C}(9)-\mathrm{C}(10)$ & $1.36(1)$ \\
\hline $\mathrm{P}(1)-\mathrm{O}(1)$ & $1.46(8)$ & $C(13)-C(14)$ & $1.41(3)$ \\
\hline $\mathrm{P}(1)-\mathrm{O}(3)$ & $1.56(1)$ & $C(15)-C(16)$ & $1.35(3)$ \\
\hline $\mathrm{P}(1)-\mathrm{O}(2)$ & $1.56(1)$ & $C(21)-C(22)$ & $1.32(2)$ \\
\hline $\mathrm{P}(1)-\mathrm{C}(11)$ & $1.79(1)$ & $\mathrm{C}(21)-\mathrm{C}(25)$ & $1.33(2)$ \\
\hline$P(3)-F(6)$ & $1.45(2)$ & $C(21)-C(26)$ & $1.57(2)$ \\
\hline $\mathrm{P}(3)-\mathrm{F}(1)$ & $1.47(2)$ & $\mathrm{C}(22)-\mathrm{C}(23)$ & $1.41(3)$ \\
\hline$P(3)-F(3)$ & $1.46(2)$ & $\mathrm{C}(22)-\mathrm{C}(27)$ & $1.51(2)$ \\
\hline$P(3)-F(4)$ & $1.47(2)$ & $\mathrm{C}(23)-\mathrm{C}(24)$ & $1.44(3)$ \\
\hline$P(3)-F(5)$ & $1.48(2)$ & $\mathrm{C}(23)-\mathrm{C}(28)$ & $1.50(1)$ \\
\hline $\mathrm{P}(3)-\mathrm{F}(2)$ & $1.49(2)$ & $C(24)-C(25)$ & $1.41(3)$ \\
\hline $\mathrm{O}(2)-\mathrm{C}(13)$ & $1.35(2)$ & $\mathrm{C}(24)-\mathrm{C}(29)$ & $1.50(2)$ \\
\hline $\mathrm{O}(3)-\mathrm{C}(15)$ & $1.48(2)$ & $\mathrm{C}(30)-\mathrm{C}(25)$ & $1.50(2)$ \\
\hline $\mathrm{O}(5)-\mathrm{C}(19)$ & $1.30(3)$ & $\mathrm{C}(20)-\mathrm{C}(19)$ & $1.45(1)$ \\
\hline $\mathrm{N}(1)-\mathrm{C}(5)$ & $1.35(9)$ & $\mathrm{O}(6)-\mathrm{C}(17)$ & $1.52(1)$ \\
\hline $\mathrm{N}(1)-\mathrm{C}(1)$ & $1.35(1)$ & $\mathrm{C}(17)-\mathrm{C}(18)$ & $1.46(1)$ \\
\hline $\mathrm{N}(2)-\mathrm{C}(10)$ & $1.34(1)$ & & \\
\hline
\end{tabular}


Table S3. Angles $\left[^{\circ}\right.$ ] for $\mathbf{C p} * \mathbf{I r P E}$

\begin{tabular}{|c|c|c|c|}
\hline $\mathrm{N}(2)-\operatorname{Ir}(1)-\mathrm{N}(1)$ & $77(2)$ & $\mathrm{C}(1)-\mathrm{N}(1)-\operatorname{Ir}(1)$ & $125(5)$ \\
\hline $\mathrm{N}(2)-\operatorname{Ir}(1)-\mathrm{C}(23)$ & $109(5)$ & $C(10)-N(2)-C(6)$ & $118(7)$ \\
\hline $\mathrm{N}(1)-\operatorname{Ir}(1)-\mathrm{C}(23)$ & $164(5)$ & $\mathrm{C}(10)-\mathrm{N}(2)-\operatorname{Ir}(1)$ & $125(6)$ \\
\hline $\mathrm{N}(2)-\operatorname{Ir}(1)-\mathrm{C}(25)$ & $126(5)$ & $\mathrm{C}(6)-\mathrm{N}(2)-\operatorname{Ir}(1)$ & $117(5)$ \\
\hline $\mathrm{N}(1)-\operatorname{Ir}(1)-\mathrm{C}(25)$ & $100(4$ & $\mathrm{N}(1)-\mathrm{C}(1)-\mathrm{C}(2)$ & $122(8)$ \\
\hline$C(23)-\operatorname{Ir}(1)-C(25)$ & $65(6)$ & $\mathrm{C}(1)-\mathrm{C}(2)-\mathrm{C}(3)$ & $121(8)$ \\
\hline $\mathrm{N}(2)-\operatorname{Ir}(1)-\mathrm{C}(21)$ & $161(5)$ & $\mathrm{C}(4)-\mathrm{C}(3)-\mathrm{C}(2)$ & $116(8)$ \\
\hline $\mathrm{N}(1)-\operatorname{Ir}(1)-\mathrm{C}(21)$ & $108(4)$ & $C(4)-C(3)-C(11)$ & $122(8)$ \\
\hline $\mathrm{C}(23)-\operatorname{Ir}(1)-\mathrm{C}(21)$ & $62(5)$ & $\mathrm{C}(2)-\mathrm{C}(3)-\mathrm{C}(11)$ & $123(8)$ \\
\hline$C(25)-\operatorname{Ir}(1)-C(21)$ & $36(6)$ & $\mathrm{C}(5)-\mathrm{C}(4)-\mathrm{C}(3)$ & $121(7)$ \\
\hline $\mathrm{N}(2)-\operatorname{Ir}(1)-\mathrm{C}(24)$ & 101(4) & $\mathrm{N}(1)-\mathrm{C}(5)-\mathrm{C}(4)$ & $121(7)$ \\
\hline $\mathrm{N}(1)-\operatorname{Ir}(1)-\mathrm{C}(24)$ & $126(6)$ & $\mathrm{N}(1)-\mathrm{C}(5)-\mathrm{C}(6)$ & $115(6)$ \\
\hline $\mathrm{C}(23)-\operatorname{Ir}(1)-\mathrm{C}(24)$ & $39(7)$ & $C(4)-C(5)-C(6)$ & $124(7)$ \\
\hline$C(25)-\operatorname{Ir}(1)-C(24)$ & $38(7)$ & $\mathrm{N}(2)-\mathrm{C}(6)-\mathrm{C}(7)$ & $121(7)$ \\
\hline $\mathrm{C}(21)-\operatorname{Ir}(1)-\mathrm{C}(24)$ & $62(5)$ & $\mathrm{N}(2)-\mathrm{C}(6)-\mathrm{C}(5)$ & $114(7)$ \\
\hline $\mathrm{N}(2)-\operatorname{Ir}(1)-\mathrm{C}(22)$ & $145(6)$ & $C(7)-C(6)-C(5)$ & $124(7)$ \\
\hline $\mathrm{N}(1)-\operatorname{Ir}(1)-\mathrm{C}(22)$ & $139(6)$ & $\mathrm{C}(6)-\mathrm{C}(7)-\mathrm{C}(8)$ & $121(8)$ \\
\hline $\mathrm{C}(23)-\operatorname{Ir}(1)-\mathrm{C}(22)$ & $38(7)$ & $\mathrm{C}(7)-\mathrm{C}(8)-\mathrm{C}(9)$ & $116(8)$ \\
\hline$C(25)-\operatorname{Ir}(1)-C(22)$ & $62(5)$ & $C(7)-C(8)-C(12)$ & $122(9)$ \\
\hline $\mathrm{C}(21)-\operatorname{Ir}(1)-\mathrm{C}(22)$ & $36(6)$ & $\mathrm{C}(9)-\mathrm{C}(8)-\mathrm{C}(12)$ & $122(9)$ \\
\hline $\mathrm{C}(24)-\operatorname{Ir}(1)-\mathrm{C}(22)$ & $64(5)$ & $\mathrm{C}(10)-\mathrm{C}(9)-\mathrm{C}(8)$ & $120(9)$ \\
\hline $\mathrm{N}(2)-\operatorname{Ir}(1)-\mathrm{Cl}(1)$ & $87(2)$ & $\mathrm{N}(2)-\mathrm{C}(10)-\mathrm{C}(9)$ & $123(9)$ \\
\hline $\mathrm{N}(1)-\operatorname{Ir}(1)-\mathrm{Cl}(1)$ & $85(2)$ & $\mathrm{C}(3)-\mathrm{C}(11)-\mathrm{P}(1)$ & $114(8)$ \\
\hline $\mathrm{C}(23)-\operatorname{Ir}(1)-\mathrm{Cl}(1)$ & $110(7)$ & $\mathrm{O}(2)-\mathrm{C}(13)-\mathrm{C}(14)$ & $116(2)$ \\
\hline $\mathrm{C}(25)-\operatorname{Ir}(1)-\mathrm{Cl}(1)$ & $148(5)$ & $\mathrm{C}(16)-\mathrm{C}(15)-\mathrm{O}(3)$ & $111(2)$ \\
\hline $\mathrm{C}(21)-\operatorname{Ir}(1)-\mathrm{Cl}(1)$ & $112(5)$ & $\mathrm{C}(22)-\mathrm{C}(21)-\mathrm{C}(25)$ & $114(2)$ \\
\hline $\mathrm{C}(24)-\operatorname{Ir}(1)-\mathrm{Cl}(1)$ & $150(6)$ & $\mathrm{C}(22)-\mathrm{C}(21)-\mathrm{C}(26)$ & $127(3)$ \\
\hline $\mathrm{C}(22)-\operatorname{Ir}(1)-\mathrm{Cl}(1)$ & $94(4)$ & $C(25)-C(21)-C(26)$ & $119(3)$ \\
\hline $\mathrm{O}(6)-\mathrm{P}(2)-\mathrm{O}(4)$ & $119(1)$ & $\mathrm{C}(22)-\mathrm{C}(21)-\operatorname{Ir}(1)$ & $73(8)$ \\
\hline $\mathrm{O}(6)-\mathrm{P}(2)-\mathrm{O}(5)$ & $106(1)$ & $\mathrm{C}(25)-\mathrm{C}(21)-\operatorname{Ir}(1)$ & $72(7)$ \\
\hline $\mathrm{O}(4)-\mathrm{P}(2)-\mathrm{O}(5)$ & $113(8)$ & $C(26)-C(21)-\operatorname{Ir}(1)$ & $131(1)$ \\
\hline $\mathrm{O}(6)-\mathrm{P}(2)-\mathrm{C}(12)$ & $103(9)$ & $\mathrm{C}(21)-\mathrm{C}(22)-\mathrm{C}(23)$ & $108(1)$ \\
\hline $\mathrm{O}(4)-\mathrm{P}(2)-\mathrm{C}(12)$ & $110(9)$ & $\mathrm{C}(21)-\mathrm{C}(22)-\mathrm{C}(27)$ & $124(2)$ \\
\hline $\mathrm{O}(5)-\mathrm{P}(2)-\mathrm{C}(12)$ & $104(8)$ & $\mathrm{C}(23)-\mathrm{C}(22)-\mathrm{C}(27)$ & $128(2)$ \\
\hline
\end{tabular}




\begin{tabular}{|c|c|c|c|}
\hline $\mathrm{O}(1)-\mathrm{P}(1)-\mathrm{O}(3)$ & $116(6)$ & $C(21)-C(22)-\operatorname{Ir}(1)$ & $72(7)$ \\
\hline $\mathrm{O}(1)-\mathrm{P}(1)-\mathrm{O}(2)$ & $116(6)$ & $C(23)-C(22)-\operatorname{Ir}(1)$ & $70(7)$ \\
\hline $\mathrm{O}(3)-\mathrm{P}(1)-\mathrm{O}(2)$ & $104(8)$ & $C(27)-C(22)-\operatorname{Ir}(1)$ & $126(1)$ \\
\hline $\mathrm{O}(1)-\mathrm{P}(1)-\mathrm{C}(11)$ & $113(6)$ & $C(22)-C(23)-C(24)$ & $106(1)$ \\
\hline $\mathrm{O}(3)-\mathrm{P}(1)-\mathrm{C}(11)$ & $105(6)$ & $C(22)-C(23)-C(28)$ & $131(3)$ \\
\hline $\mathrm{O}(2)-\mathrm{P}(1)-\mathrm{C}(11)$ & $101(5)$ & $C(24)-C(23)-C(28)$ & $123(3)$ \\
\hline $\mathrm{F}(6)-\mathrm{P}(3)-\mathrm{F}(1)$ & $87(2)$ & $C(22)-C(23)-\operatorname{Ir}(1)$ & $72(7)$ \\
\hline$F(6)-P(3)-F(3)$ & $90(2)$ & $C(24)-C(23)-\operatorname{Ir}(1)$ & $71(7)$ \\
\hline $\mathrm{F}(1)-\mathrm{P}(3)-\mathrm{F}(3)$ & $176(2)$ & $C(28)-C(23)-\operatorname{Ir}(1)$ & $128(9)$ \\
\hline $\mathrm{F}(6)-\mathrm{P}(3)-\mathrm{F}(4)$ & $98(2)$ & $C(25)-C(24)-C(23)$ & $106(1)$ \\
\hline$F(1)-P(3)-F(4)$ & $93(2)$ & $C(25)-C(24)-C(29)$ & $117(2)$ \\
\hline $\mathrm{F}(3)-\mathrm{P}(3)-\mathrm{F}(4)$ & $85(1)$ & $C(23)-C(24)-C(29)$ & $136(2)$ \\
\hline $\mathrm{F}(6)-\mathrm{P}(3)-\mathrm{F}(5)$ & $168(2)$ & $C(25)-C(24)-\operatorname{Ir}(1)$ & $70(6)$ \\
\hline $\mathrm{F}(1)-\mathrm{P}(3)-\mathrm{F}(5)$ & $88(2)$ & $C(23)-C(24)-\operatorname{Ir}(1)$ & $70(7)$ \\
\hline $\mathrm{F}(3)-\mathrm{P}(3)-\mathrm{F}(5)$ & $95(2)$ & $C(29)-C(24)-\operatorname{Ir}(1)$ & $126(1)$ \\
\hline $\mathrm{F}(4)-\mathrm{P}(3)-\mathrm{F}(5)$ & $93(2)$ & $C(21)-C(25)-C(24)$ & 107(1) \\
\hline $\mathrm{F}(6)-\mathrm{P}(3)-\mathrm{F}(2)$ & $91(2)$ & $C(21)-C(25)-C(30)$ & $126(2)$ \\
\hline $\mathrm{F}(1)-\mathrm{P}(3)-\mathrm{F}(2)$ & $98(1)$ & $C(24)-C(25)-C(30)$ & $126(2)$ \\
\hline $\mathrm{F}(3)-\mathrm{P}(3)-\mathrm{F}(2)$ & $85(1)$ & $C(21)-C(25)-\operatorname{Ir}(1)$ & $72(8)$ \\
\hline $\mathrm{F}(4)-\mathrm{P}(3)-\mathrm{F}(2)$ & $166(2)$ & $C(24)-C(25)-\operatorname{Ir}(1)$ & 71(7) \\
\hline $\mathrm{F}(5)-\mathrm{P}(3)-\mathrm{F}(2)$ & $79(2)$ & $C(30)-C(25)-\operatorname{Ir}(1)$ & $128(1)$ \\
\hline $\mathrm{C}(13)-\mathrm{O}(2)-\mathrm{P}(1)$ & $130(1)$ & $\mathrm{C}(8)-\mathrm{C}(12)-\mathrm{P}(2)$ & $116(1)$ \\
\hline $\mathrm{C}(15)-\mathrm{O}(3)-\mathrm{P}(1)$ & $129(1)$ & $\mathrm{O}(5)-\mathrm{C}(19)-\mathrm{C}(20)$ & $125(3)$ \\
\hline $\mathrm{C}(19)-\mathrm{O}(5)-\mathrm{P}(2)$ & $131(2)$ & $\mathrm{P}(2)-\mathrm{O}(6)-\mathrm{C}(17)$ & $123(2)$ \\
\hline$C(5)-N(1)-C(1)$ & $119(7)$ & $\mathrm{C}(18)-\mathrm{C}(17)-\mathrm{O}(6)$ & $154(3)$ \\
\hline $\mathrm{C}(5)-\mathrm{N}(1)-\operatorname{Ir}(1)$ & $117(5)$ & & \\
\hline
\end{tabular}




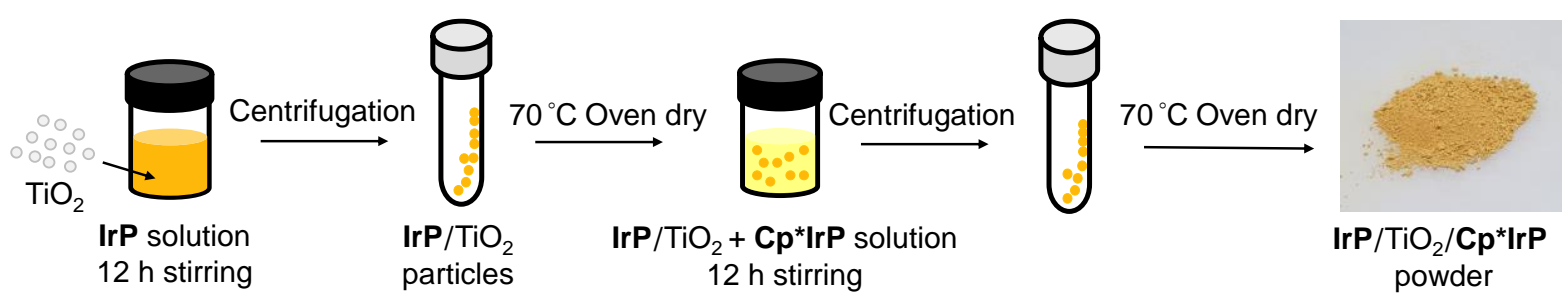

Figure S8. Detailed procedure for preparation of $\mathbf{I r P} / \mathrm{TiO}_{2} / \mathbf{C p} * \mathbf{I r P}$ ternary sample. 


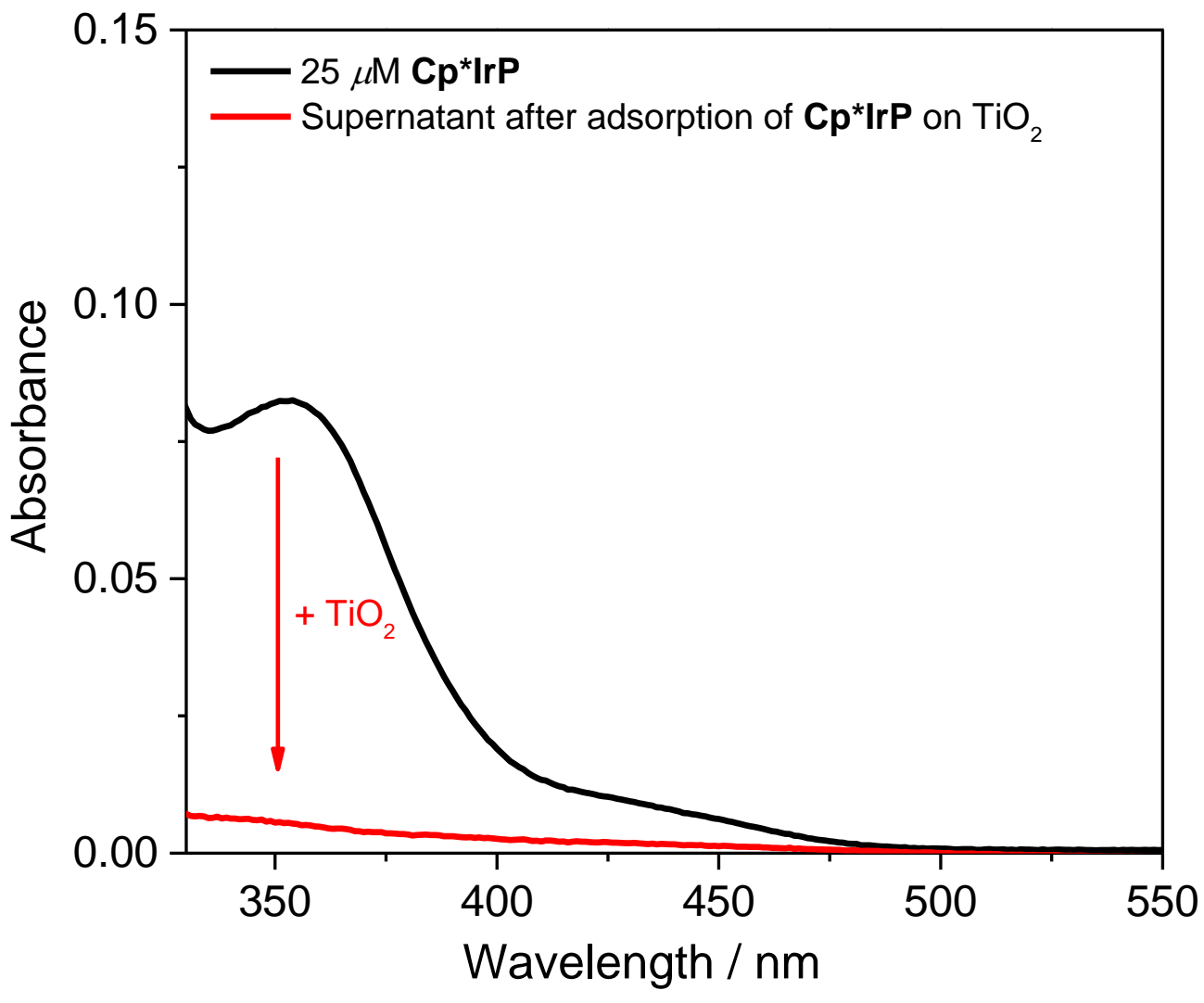

Figure S9. Comparable absorption spectra of EtOH solution of $\mathbf{C p} * \mathbf{I r P}$ before (black line) and after (red line) adsorption process of $\mathbf{C p} * \mathbf{I r P}$ with $\mathrm{TiO}_{2}$ particles. 


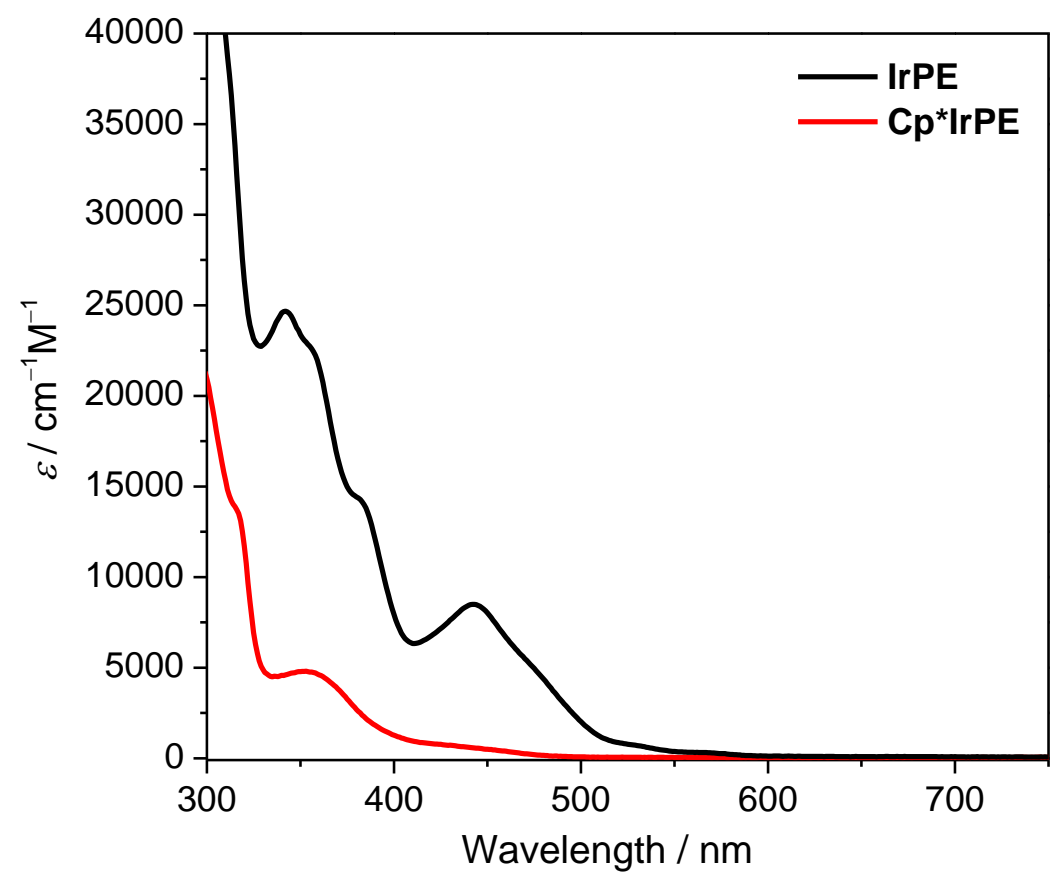

Figure S10. UV-vis absorption spectra of $\operatorname{IrPE}(10 \mu \mathrm{M})$ and $\mathbf{C p} * \operatorname{IrPE}(10 \mu \mathrm{M})$ in DMF. 

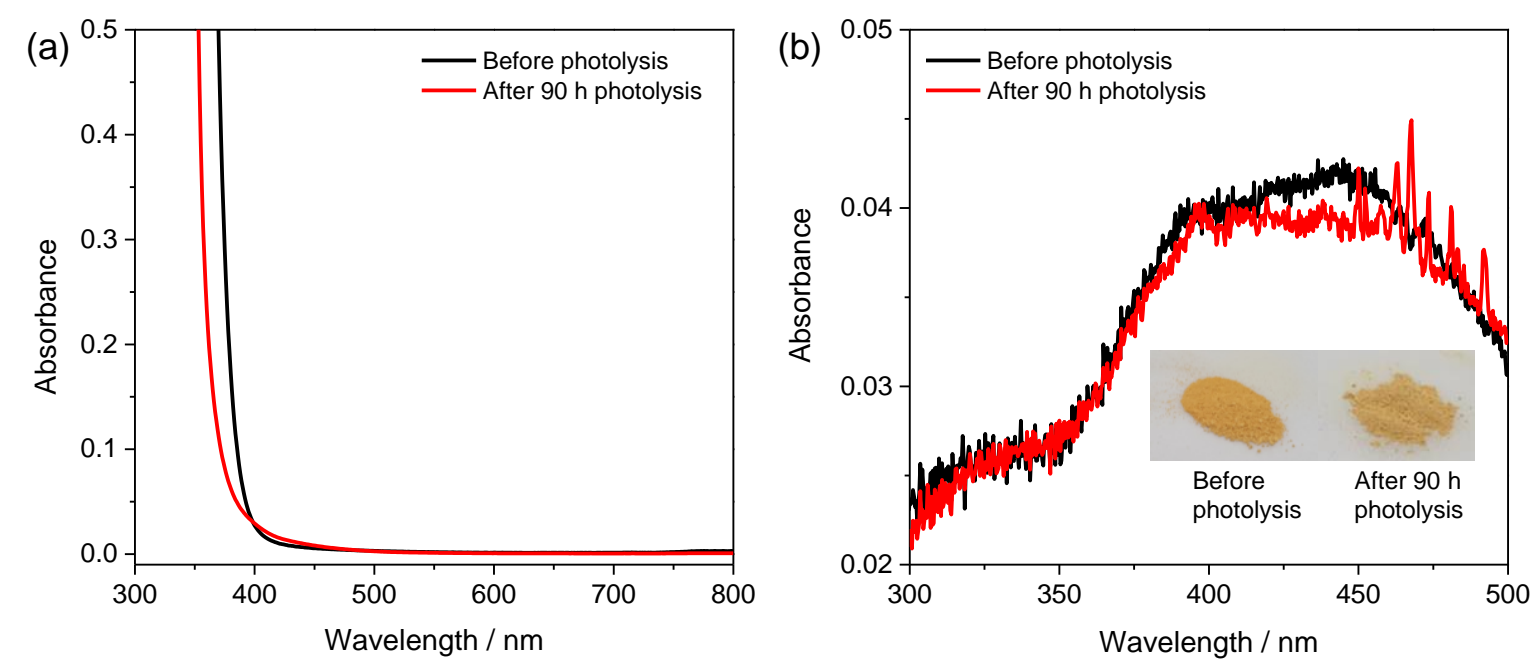

Figure S11. UV-vis absorption spectrum of (a) the filtrate and (b) heterogeneous residue obtained before (black line) and after (red line) $90 \mathrm{~h}$ photolysis of hetero-ternary sample containing $0.1 \mathrm{M} \mathrm{BIH}$, and $10 \mathrm{mg} \operatorname{IrP}(1$ $\mu \mathrm{mol}) / \mathrm{TiO}_{2}(10 \mathrm{mg}) / \mathbf{C} \mathbf{p}^{*} \operatorname{IrP}(0.1 \mu \mathrm{mol})$ particles in $2 \mathrm{~mL} \mathrm{DMF}-d_{7} / \mathrm{TEOA}(16.7 \mathrm{vol} \%$ TEOA) solution; irradiation at $>500 \mathrm{~nm}$. To trace the fate of $\operatorname{Ir}(\mathrm{III})$ photosensitizer (IrP) anchored to the $\mathrm{TiO}_{2}$ surface, two $\operatorname{IrP} / \mathrm{TiO}_{2} / \mathbf{C p}$ *IrP samples before and after $90 \mathrm{~h}$ photolysis were put in a homemade integrating sphere absorption spectroscopy tool (see Figure S1). In each measurement, the almost similarity in absorption feature and visual photoimage (see inset of Figure S11b) between $\mathbf{I r P} / \mathrm{TiO}_{2} / \mathbf{C p}$ *IrP powders and the reaction solutions filtered before and after $90 \mathrm{~h}$ photolysis indicates that the anchored $\mathbf{I r P}$ units remain on $\mathrm{TiO}_{2}$ without its significant chemical change during photolysis. 


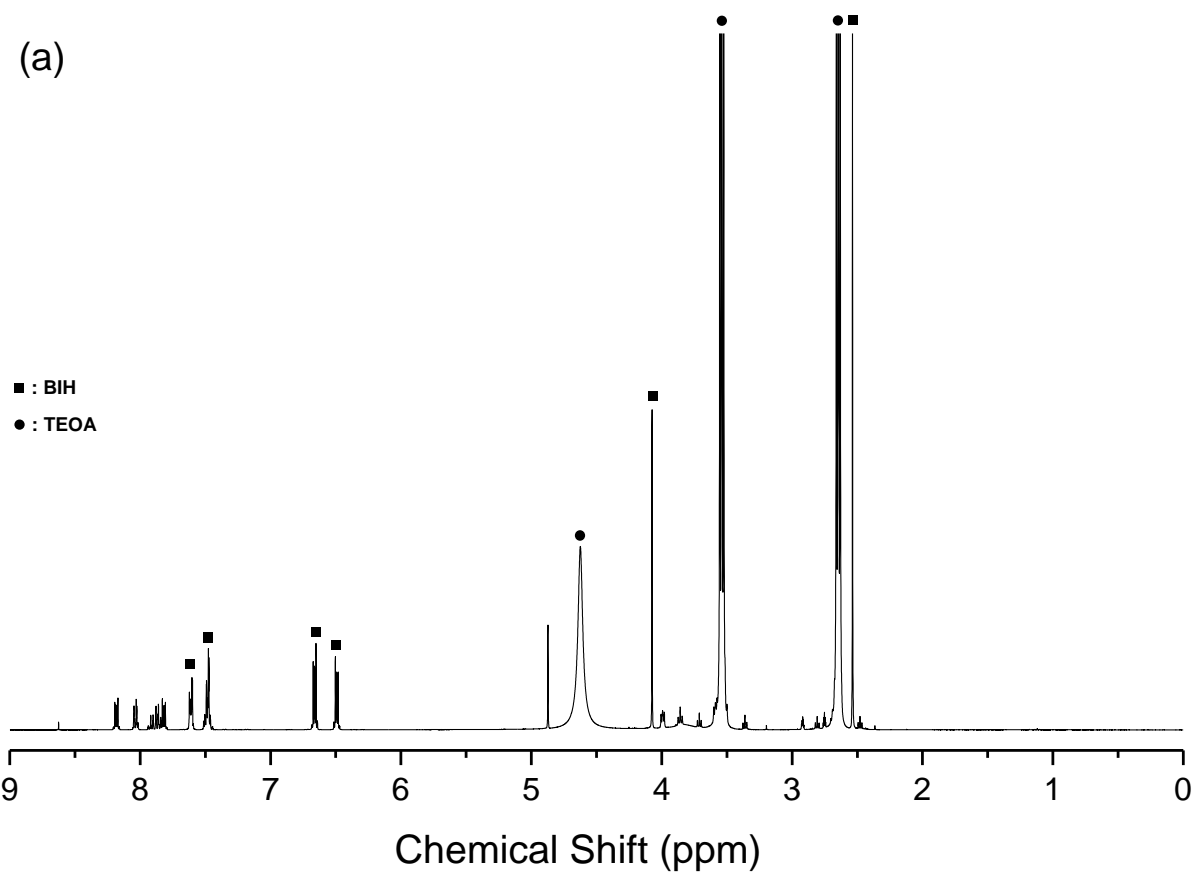

(b)

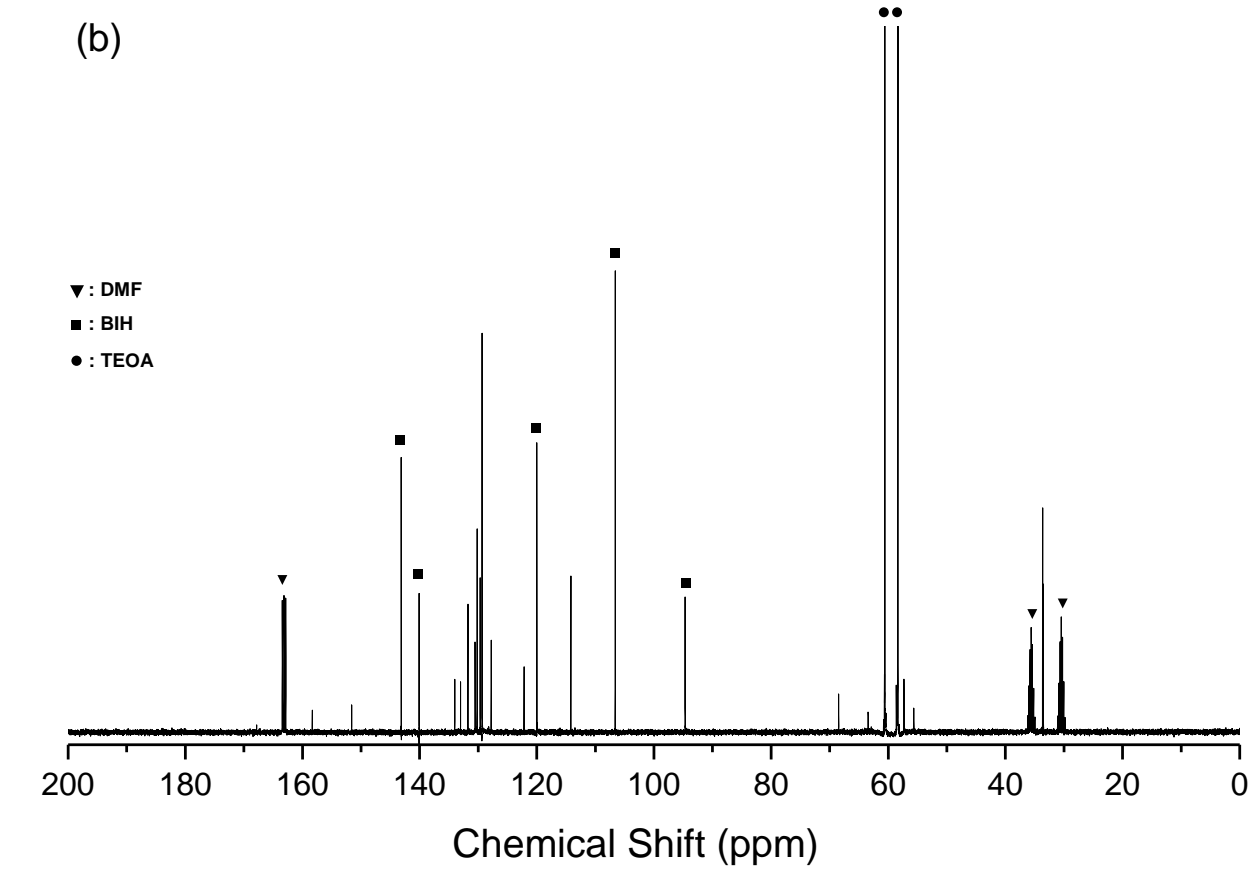

Figure S12. ${ }^{1} \mathrm{H}$ NMR (a) and ${ }^{13} \mathrm{C}$ NMR (b) spectrum of reaction solution filtered after $90 \mathrm{~h}$ photolysis of heteroternary sample containing $0.1 \mathrm{M} \mathrm{BIH}$, and $10 \mathrm{mg} \mathrm{IrP/TiO} 2 / \mathbf{C p}$ *IrP particles in $2 \mathrm{~mL}$ DMF- $d_{7} / \mathrm{TEOA}(16.7 \mathrm{vol} \%$ TEOA) solution; irradiation at $>500 \mathrm{~nm}$. The symbols $\mathbf{\nabla}, \mathbf{m}$, and $\bullet$ represent DMF, BIH, and TEOA, respectively. To identify the presence of $\mathbf{C p}$ *IrP catalyst desorbed from $\mathrm{TiO}_{2}$ during photolysis, we performed the comparison of ${ }^{1} \mathrm{H} /{ }^{13} \mathrm{C}$ NMR spectra before and after $90 \mathrm{~h}$ photoreaction. In the both NMR spectrum, no appearance of methyl peak of $\mathrm{Cp}^{*}$ ligand (1.68 ppm in ${ }^{1} \mathrm{H}$ NMR and $8.1 \mathrm{ppm}$ in ${ }^{13} \mathrm{C} \mathrm{NMR}$ ), which is a characteristic of $\mathbf{C} \mathbf{p}^{*} \mathbf{I r P}$ complex, proves that a desorption of loaded $\mathbf{C}$ * $*$ IrP complex did not occur during $90 \mathrm{~h}$ photolysis. 

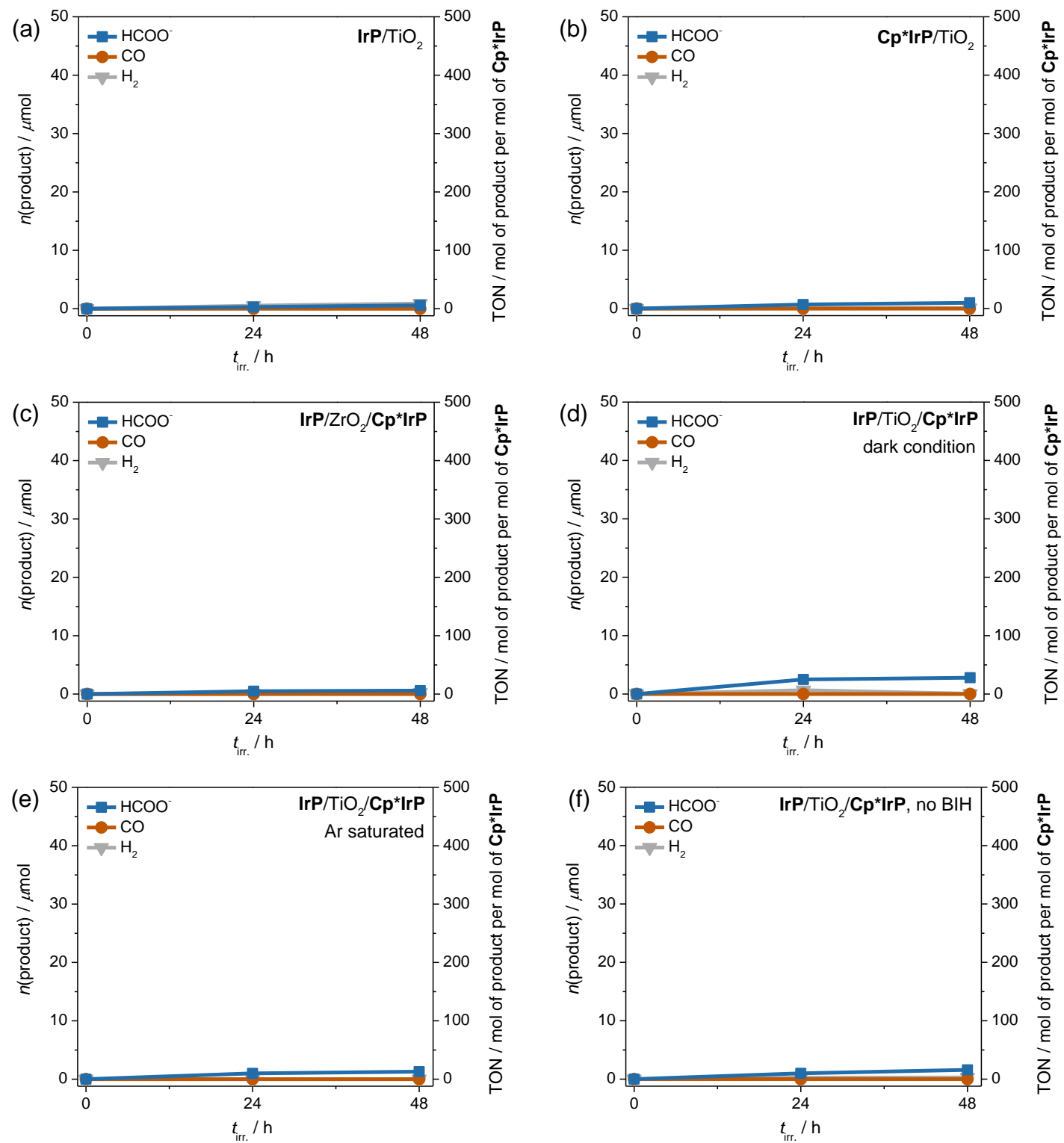

Figure S13. Plots of formate/CO production versus irradiation time for control samples (10 mg): (a) $\mathbf{I r P}(1$ $\mu \mathrm{mol}) / \mathrm{TiO}_{2}(10 \mathrm{mg})$, (b) $\mathrm{TiO}_{2}(10 \mathrm{mg}) / \mathbf{C p} * \mathbf{I r P}(0.1 \mu \mathrm{mol})$, and (c) $\mathbf{I r P}(1 \mu \mathrm{mol}) / \mathrm{ZrO}_{2}(10 \mathrm{mg}) / \mathbf{C p} * \mathbf{I r P}(0.1 \mu \mathrm{mol})$ in $\mathrm{CO}_{2}$-saturated mixed DMF/TEOA (16.7 vol \% TEOA) solution containing $0.1 \mathrm{M} \mathrm{BIH}$; irradiation at $\lambda>500 \mathrm{~nm}$ at 298 K. (d) Under dark. (e) In Ar atmosphere. (f) Without BIH. 


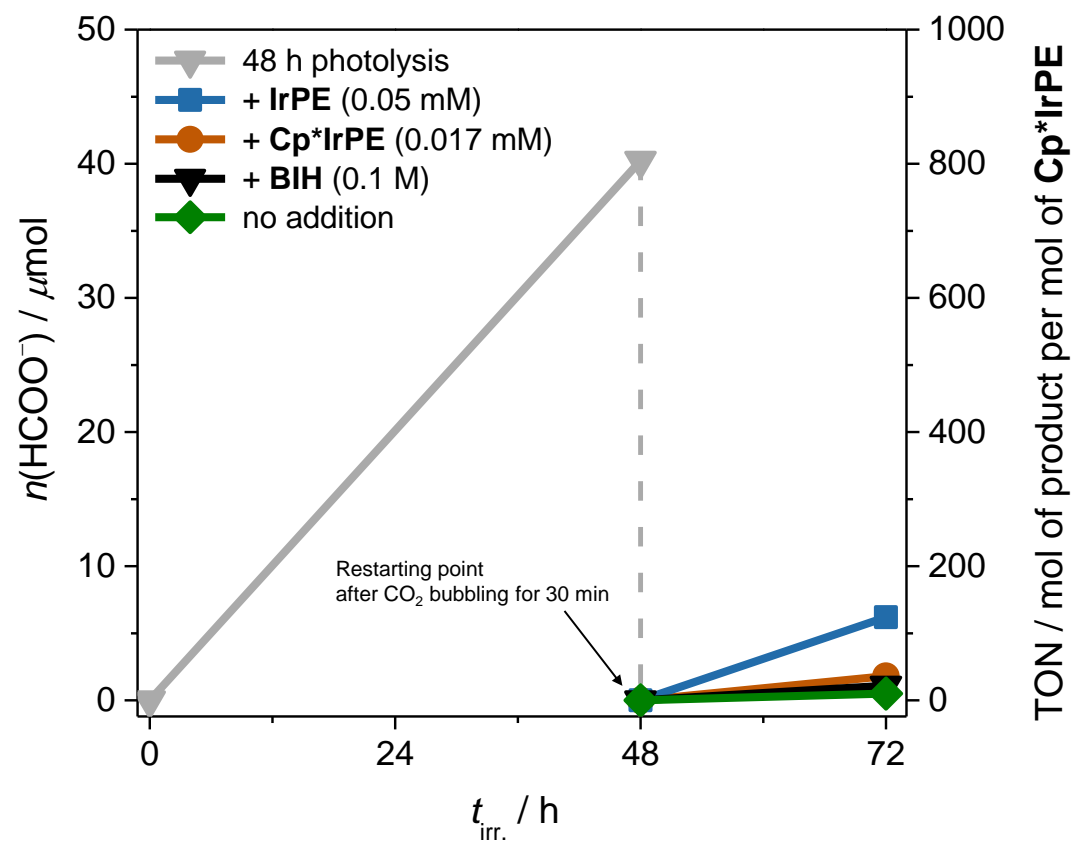

Figure S14. Formation of formate in photolysis restarted with re-addition of different components $(0.05 \mathrm{mM}$ IrPE, $0.017 \mathrm{mM} \mathrm{Cp} * \mathbf{I r P E}$, or $0.1 \mathrm{M} \mathrm{BIH})$ after $48 \mathrm{~h}$ photolysis of homogeneous sample $(0.05 \mathrm{mM}$ IrPE $+0.017 \mathrm{mM}$ Cp*IrPE) in $3 \mathrm{~mL}$ of $\mathrm{CO}_{2}$-saturated DMF/TEOA mixture solvent (16.7 vol \% TEOA) containing $0.1 \mathrm{M} \mathrm{BIH}$; irradiation at $>500 \mathrm{~nm}$. 

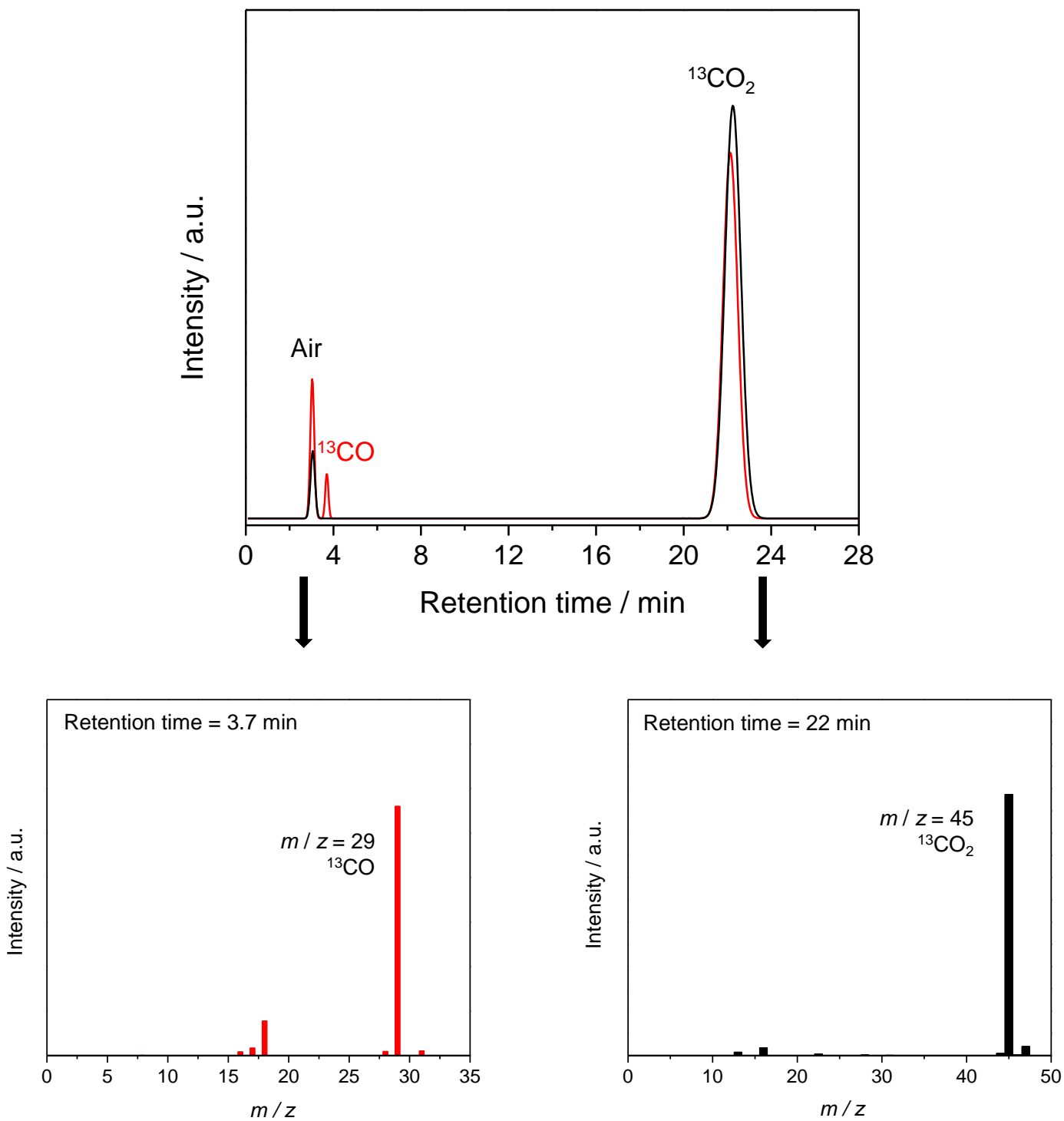

Figure S15. GC spectrum of gas in the reation vessel before (black line) and after (red line) photoreaction and MS spectra of each retention time: GC-MS spectra was measured by Agilent Technologies 7890A GC equipped with 5975C inert MSD with Triple-Axis detector using a SUPELCO CarboxenTM 1010 PLOT Fused Silica Capillary column. 

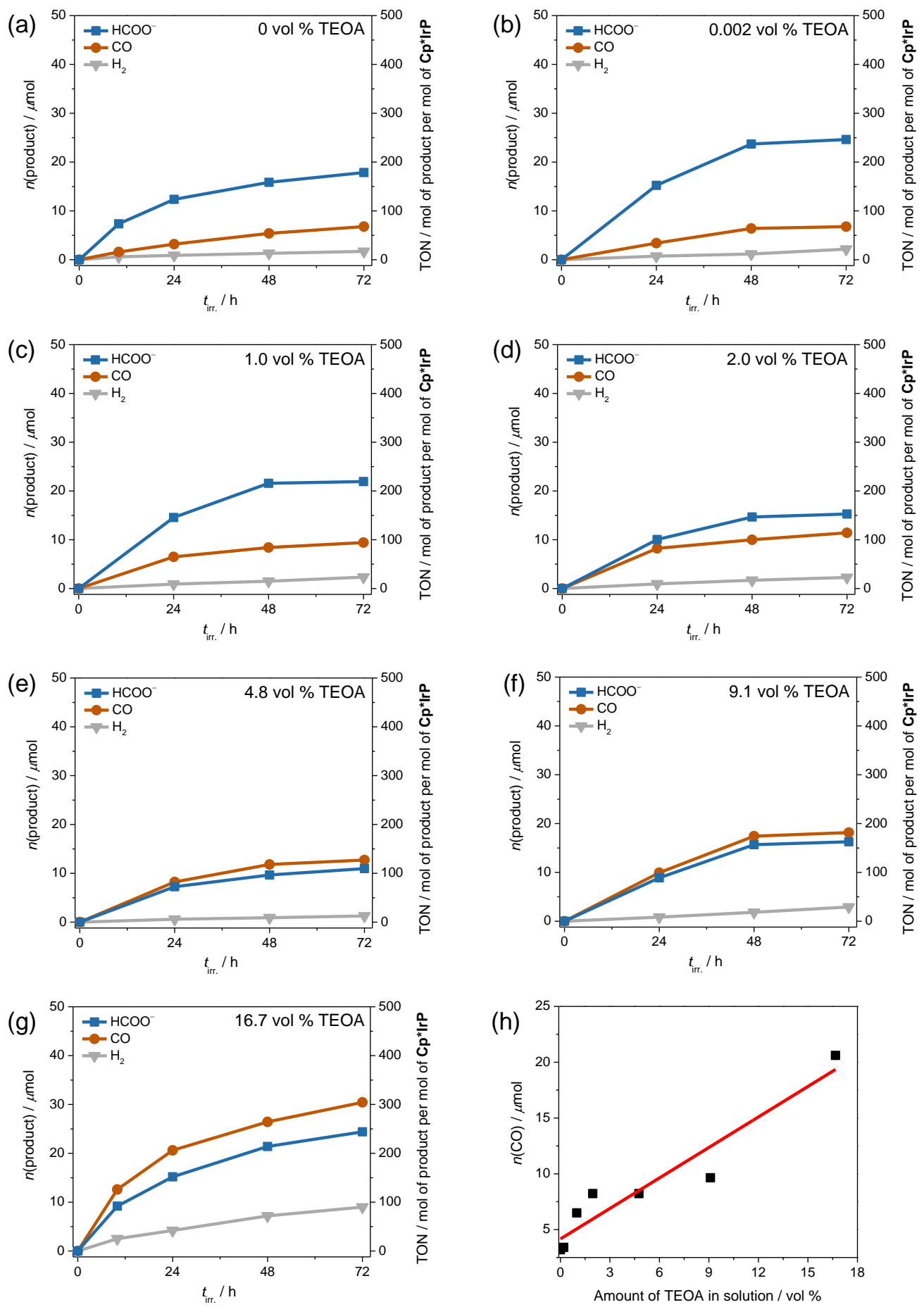

Figure S16. Plots of formate and CO formation versus irradiation time for ternary hybrid system ( $\operatorname{IrP}(1$ $\left.\mu \mathrm{mol}) / \mathrm{TiO}_{2}(10 \mathrm{mg}) / \mathbf{C} \mathbf{p}^{*} \operatorname{IrP}(0.1 \mu \mathrm{mol})\right)$ in the absence of TEOA (a) and in the presence of TEOA additive $(x$ vol \%) $(x=0.002(\mathrm{~b}), 1.0(\mathrm{c}), 2.0(\mathrm{~d}), 4.8(\mathrm{e}), 9.1(\mathrm{f})$, and $16.7(\mathrm{~g}))$ in $\mathrm{CO}_{2}$-saturated DMF containing 0.1 M BIH; irradiation at $>500 \mathrm{~nm}$. (h) Plots of CO production ( $\mu \mathrm{mol}$ ) after $24 \mathrm{~h}$ photoreaction versus vol \% of TEOA in total volume of reaction solvent. 
Table S4. Results of visible-ligth-driven $\mathrm{CO}_{2}$ reduction with variation of TEOA content ( 0 to 16.7 vol $\left.\%\right)^{a}$

\begin{tabular}{|c|c|c|c|c|c|c|}
\hline \multirow{2}{*}{ entry } & \multirow{2}{*}{ vol \% TEOA } & \multicolumn{2}{|c|}{ formate } & \multicolumn{2}{|c|}{$\mathrm{CO}$} & \multirow{2}{*}{$\frac{\mathrm{H}_{2}}{\mu \mathrm{mol}}$} \\
\hline & & TON & $\mu \mathrm{mol}$ & TON & $\mu \mathrm{mol}$ & \\
\hline 1 & $w / o$ & 178 & 17.8 & 68 & 6.8 & 1.7 \\
\hline 2 & 0.002 & 246 & 24.6 & 68 & 6.8 & 2.2 \\
\hline 3 & 1.0 & 219 & 21.9 & 94 & 9.4 & 2.3 \\
\hline 4 & 2.0 & 153 & 15.3 & 114 & 11.4 & 2.3 \\
\hline 5 & 4.8 & 110 & 11.0 & 127 & 12.7 & 1.3 \\
\hline 6 & 9.1 & 162 & 16.2 & 182 & 18.2 & 2.9 \\
\hline 7 & 16.7 & 244 & 24.4 & 304 & 30.4 & 9.0 \\
\hline
\end{tabular}

${ }^{a} \mathbf{I r P}(1 \mu \mathrm{mol}) / \mathrm{TiO}_{2}(10 \mathrm{mg}) / \mathbf{C p} * \operatorname{IrP}(0.1 \mu \mathrm{mol})$ in $\mathrm{CO}_{2}$-saturated DMF/TEOA mixture solvent $(0$ to 16.7 vol \% TEOA) containing $0.1 \mathrm{M} \mathrm{BIH}$; irradiation at $>500 \mathrm{~nm}$. See the detailed photocatalysis conditions in the Experimental Section. 

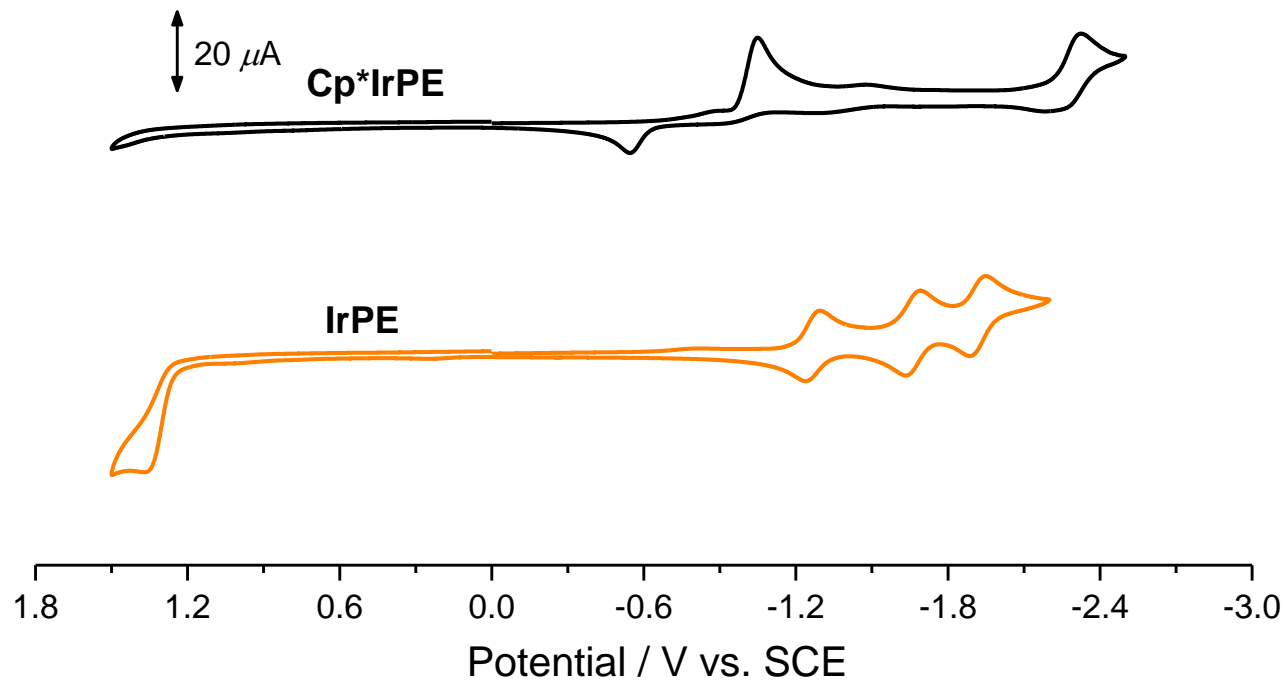

Figure S17. Cyclic voltammograms of IrPE and Cp*IrPE (1.0 mM) in DMF in the presence of 0.10 M TBAP, scan rate at $100 \mathrm{mV} \mathrm{s}^{-1}$.

Table S5. Electrochemical properties of IrPE and Cp*IrPE used in this study ${ }^{a}$

\begin{tabular}{lcccc}
\hline Compound & $E_{\mathrm{pa}}{ }^{\text {red }}[\mathrm{V}]$ & $E_{\mathrm{pc}}{ }^{\text {red }}[\mathrm{V}]$ & $E_{1 / 2}{ }^{\text {red }}[\mathrm{V}]$ & $E_{\mathrm{pa}}{ }^{\text {ox }}[\mathrm{V}]$ \\
\hline Cp*IrPE & $-0.55,-2.22$ & $-1.06,-2.33$ & $-0.81,-2.28$ & n.d. \\
\hline IrPE & $-1.24,-1.63,-1.88$ & $-1.28,-1.69,-1.95$ & $-1.26,-1.66,-1.92$ & 1.33 \\
\hline${ }^{a}$ Condition: a glassy carbon (dia. $2.0 \mathrm{~mm}$ ), platinum wire, and $\mathrm{Ag} / \mathrm{AgNO}$ electrode containing Ar-degassed DMF electrolyte solution (TBAP, \\
0.1 M) behind a Vycor tip were used as the working, counter, and reference electrodes, respectively. The potential values were calibrated \\
versus the ferrocene/ferrocenium $\left(\mathrm{Fc} / \mathrm{Fc}^{+}\right.$) redox couple, and then corrected to the saturated calomel electrode (SCE) on the basis of an $\mathrm{Fc} \mid \mathrm{Fc}^{+}$ \\
redox potential of $0.38 \mathrm{~V}$ versus SCE for direct comparison with the literature values.
\end{tabular}

